(2) Open Access Full Text Article

\title{
Upregulated long noncoding RNA PANDAR predicts an unfavorable prognosis and promotes tumorigenesis in cholangiocarcinoma
}

This article was published in the following Dove Press journal:

OncoTargets and Therapy

6 June 2017

Number of times this article has been viewed

Yi X $u^{1,2}$

Xingming Jiang'

Yunfu Cui'

'Department of Hepatopancreatobiliary Surgery, Second Affiliated Hospital of Harbin Medical University, ${ }^{2}$ The Key Laboratory of Myocardial Ischemia, Harbin Medical University, Ministry of Education, Harbin, People's Republic of China
Correspondence: Yunfu Cui;

Xingming Jiang

Department of Hepatopancreatobiliary Surgery, Second Affiliated Hospital of Harbin Medical University, No 246 Xuefu Road, Harbin, Heilongjiang I50086,

People's Republic of China

Email yfcui777@hotmail.com;

xmjiang@hrbmu.edu.cn
Abstract: Cholangiocarcinoma (CCA) is one of the most malignant human cancers with increasing incidence worldwide. LncRNAs have emerged as gene regulators and prognostic biomarkers in a variety of neoplasms. PANDAR, a novel cancer-related lncRNA, has been reported to be upregulated in diverse human carcinomas. In this study, we aimed to investigate the clinical significance of lncRNA PANDAR in CCA and explore its functional roles in CCA cells including cell proliferation, apoptosis, migration, invasion and epithelial-to-mesenchymal transition (EMT). The results showed that PANDAR was significantly upregulated in CCA tissue specimens and cell lines, and its high expression was closely associated with lymph node invasion $(P=0.004)$, TNM stage $(P=0.034)$ and postoperative relapse $(P=0.006)$ in patients with CCA. Thus, overexpression of PANDAR could serve as an independent prognostic biomarker of CCA. Furthermore, silencing of PANDAR followed by siRNA significantly inhibited cell proliferation and increased apoptosis in CCA cells. In addition, suppression of PANDAR impaired migration and invasion capacity in vitro partly by affecting EMT. Overall, our findings showed that lncRNA PANDAR serves as a novel prognostic biomarker and therapeutic target for CCA.

Keywords: cholangiocarcinoma, lncRNA, PANDAR, prognosis, tumorigenesis

\section{Introduction}

Cholangiocarcinoma (CCA) originates from the epithelium of hepatic biliary trees with limited therapeutic options and poor prognosis. ${ }^{1}$ According to epidemiological data, there is an increasing incidence of CCA worldwide over the past decades, and currently, radical resection is the only treatment option available. ${ }^{2,3}$ However, due to the early invasive and metastatic characteristics of CCA, most patients are diagnosed at the advanced stages and are ineligible for surgical intervention. In addition, surgical resection is associated with a high rate of recurrence, and the 5-year survival rate is less than $10 \%$. Moreover, CCA is also insensitive to palliative treatments including chemotherapy and radiotherapy. 4,5 Therefore, exploring the underlying mechanisms of tumorigenesis and cancer progression, in addition to identifying new therapeutic targets and novel biomarkers associated with diagnosis/prognosis, is very important.

With the progression of whole-genome sequencing technology, it has been found that less than $2 \%$ of the mammalian genome is in protein-encoded regions and the rest is in noncoding RNAs. ${ }^{6}$ LncRNAs, imperative members of noncoding RNA family, are transcripts of at least 200 nucleotides in length and have no or limited protein-coding potential. ${ }^{7}$ Recently, many lncRNAs have been reported to play imperative roles in 
tumorigenesis, cancer progression and drug resistance. ${ }^{8-11}$ LncRNAs exert a large number of molecular functions, such as modulation of alternative splicing, chromatin remodeling and RNA metabolism. ${ }^{12-14}$ They function mostly by binding with miRNAs as sponges or interacting with proteins. However, studies exploring the functional role of lncRNAs in CCA are very scarce. ${ }^{15}$

PANDAR, a novel lncRNA consisting of 1506 nucleotides in length and mapped to $6 \mathrm{p} 21.2$, was originally discovered in a p53-dependent manner and interacts with the transcription factor NF-YA. NF-YA is linked to the expression of proapoptotic genes, which has been proved by chromatin immunoprecipitation method. ${ }^{16}$ Recently, emerging evidence indicates that PANDAR is upregulated and involved in carcinogenesis and progression of various neoplasms including gastric cancer, hepatocellular carcinoma, bladder cancer and colorectal cancer. ${ }^{17-20}$ However, the biological role and clinical relevance of PANDAR in CCA remains to be elucidated.

In this study, we aimed to characterize the expression of PANDAR in CCA and their corresponding adjacent noncancerous tissues and evaluate the association of PANDAR with clinicopathological features of CCA. Furthermore, we explored the functional roles of PANDAR in CCA through examining proliferation, apoptosis, migration, invasion and epithelial-tomesenchymal transition (EMT) after silencing PANDAR.

\section{Materials and methods}

\section{Ethics statement}

This study was performed with the approval of the Ethics Review Committee of Harbin Medical University (KY2016015, approval date, 2016.3.1), and all the recruited patients signed informed consent before participating in this study.

\section{Patients and tissue samples}

In this study, we collected 67 pairs of CCA tumor and matched nontumor tissue samples at the Second Affiliated Hospital of Harbin Medical University from 2011 to 2013. Tissue specimens after surgery were collected and immediately stored in liquid nitrogen until required. All recruited patients did not receive chemotherapy or radiotherapy preoperatively, and the diagnoses were confirmed by histological examination.

\section{RNA extraction and quantitative real- time PCR (qRT-PCR)}

Total RNA of the tissues and cells was extracted by Trizol reagent (Thermo Fisher Scientific, Waltham, MA, USA) according to the manufacturer's recommendation and then converted to cDNA using a reverse transcription kit (Roche, Germany). We performed qRT-PCR by using FastStart Universal SYBR Green Master (Roche Applied Science, Mannheim, Germany) and a BIO-RAD C1000 Thermal Cycler. The following primer sets for PANDAR and GAPDH were used: PANDAR primers forward: 5'-CTGTTAAGGTGGTGGCATTG-3', reverse: 5'-GGAGGCTCATACTGGCTGAT-3'; GAPDH primers forward: 5'-GGGAGCCAAAAGGGTCAT-3', reverse: 5'-GAGTCCTTCCACGATACCAA-3'. The relative expression was calculated by delta-delta $\mathrm{CT}$ method.

\section{Cell culture and siRNA transfection}

HCCC-9810 and RBE cells used in the study were purchased from the Cell Bank of Type Culture of Chinese Academy of Sciences (Shanghai, People's Republic of China). The other CCA cell lines including QBC939, Huh-28, HuCCT1, KMBC and CCLP-1 and human intrahepatic biliary epithelial cell (HIBEC) were preserved in our laboratory. The cells were cultivated in DMEM or RPMI-1640 supplemented with 10\% FBS (Thermo Fisher Scientific) in a $37^{\circ} \mathrm{C}$ humidified incubator with $5 \% \mathrm{CO}_{2}$. All of the cells were passaged for no more than 6 months. The siRNA specifically targeting PANDAR (si-PANDAR) and a scrambled nucleotide (si-NC) were commercially constructed by GenePharma Corporation (Shanghai, People's Republic of China). The cells cultured in plates were allowed to grow to half confluence prior to transfection with corresponding siRNA using Lipofectamine 3000 transfection reagent (Thermo Fisher Scientific) in serum-free medium. Flow cytometry was used to detect the fluorescently labeled siRNAs and examine the transfection efficiency. After $48 \mathrm{~h}$, cell lysates were harvested for qRT-PCR and western blot analysis. The target sequences of si-PANDAR are as follows: si-PANDAR-1: 5'-AAUGUGUGCACGUAACAGAUU-3', si-PANDAR-2: 5'-GGGCAUGUUUUCACAGAGGUU-3', si-PANDAR-3: 5'-GCAAUCUACAACCUGUCUU-3'.

\section{Cell proliferation assays}

Cell proliferation was determined using Cell Counting Kit-8 (CCK-8) (Dojindo, Japan) according to the manufacturer's instruction. Briefly, QBC939 and RBE cells transfected with siRNA targeting PANDAR or negative control were seeded in 96-well flat-bottomed plates at a density of 1,500 cells/well. At each monitored time (0, 24, 48, 72 and $96 \mathrm{~h}), 10 \mu \mathrm{L}$ CCK-8 reagent was added into the corresponding wells and incubated at $37^{\circ} \mathrm{C}$ for $2 \mathrm{~h}$ prior to reading absorbance at $450 \mathrm{~nm}$ by using a microplate reader (Tecan, Männedorf, Switzerland). For the clonogenic assay, a certain number of cells transfected with si-PANDAR or si-NC were trypsinized into a single-cell suspension and planted in each well of a $3.5 \mathrm{~cm}$ dish and 
maintained in proper media supplemented with 10\% FBS for about 14 days until there were visible colonies formed. After fixation with paraformaldehyde and staining with $0.1 \%$ crystal violet (Beyotime, Haimen, People's Republic of China), number of colonies were counted from three randomly chosen fields under a light microscope.

\section{Cell apoptosis assays}

QBC939 and RBE cells transfected with corresponding siRNA in the exponential growth phase were trypsinized and washed twice with cold PBS, followed by resuspension in the provided binding buffer. After being labeled with $5 \mu \mathrm{L}$ Annexin V and $5 \mu \mathrm{L}$ propidium iodide (BD Biosciences, San Jose, CA, USA) and incubated at room temperature for $15 \mathrm{~min}$ according to the manufacturer's instructions, the treated cells were then detected by flow cytometry (FACScan, BD Biosciences). Cell apoptosis was also determined by acridine orange/ethidium bromide (AO/EB) double fluorescence staining analysis. AO instantly permeates intact cell membrane and stains the nucleus with green fluorescence. EB stains DNA in orange or red fluorescence when the cell membrane is broken. Thus, $\mathrm{AO} / \mathrm{EB}$ staining helps to differentiate live cells from apoptotic cells. In brief, after $48 \mathrm{~h}$ transfection, cells were stained with prepared AO/EB mixing solution for 5 min (Solarbio, Beijing, People's Republic of China) before observation under a fluorescence microscope.

\section{Relative caspase activity determination}

Caspase-3 and caspase-9 were introduced to evaluate siPANDAR induced apoptosis in this study. The relative expression of caspase- 3 and caspase- 9 was measured using the Caspase-3 Activity Kit and Caspase-9 Activity Kit (Solarbio). In brief, proteins were lysed from siRNA-treated cells after $48 \mathrm{~h}$ and then were added into 96-well plates with $90 \mu \mathrm{L}$ of the reaction buffer and caspase substrate $(2 \mathrm{mM})$. After incubation at $37^{\circ} \mathrm{C}$ for $4 \mathrm{~h}$, caspase activity was measured by a microplate reader at $405 \mathrm{~nm}$.

\section{Wound healing assay}

QBC939 and RBE cells transfected with si-PANDAR and their corresponding control cells were plated in 6-well plates and were grown up to a confluency of $70 \%-80 \%$. Straight wounds were created by scraping the cells with a $200 \mu \mathrm{L}$ pipette tip and the cell debris was cleaned up. Photographs of wound closure area were captured $0 \mathrm{~h}$ and $36 \mathrm{~h}$ after wound formation.

\section{Migration and invasion assays}

The cell motility assay was performed by using 24-well transwell chamber (Costar, Washington, DC, USA) with or without $40 \mu \mathrm{L}$ Matrigel (BD Biosciences) coating. Transfected cells $\left(5 \times 10^{4}\right.$ cells/well $)$ were suspended in $0.2 \mathrm{~mL}$ serum-free medium prior to the addition of upper chamber of inserts in the 24-well plate. The lower compartment was filled with $0.6 \mathrm{~mL}$ of complete medium. After $24 \mathrm{~h}$ incubation, the cells migrated or invaded through membrane were fixed with paraformaldehyde and were stained with $0.1 \%$ crystal violet. Three randomly high-power fields were counted. Experiments were performed in triplicate.

\section{Western blot assay}

After $48 \mathrm{~h}$ transfection, QBC939 and RBE cells were lysed in RIPA buffer with protease inhibitors (Beyotime). Protein concentration was measured by the bicinchoninic acid method (Beyotime). Equal amounts of proteins were electrophoresed on sodium dodecyl sulfate polyacrylamide gel before being transferred to polyvinylidene fluoride membranes (GE Healthcare, Milwaukee, WI, USA). Afterwards, $5 \%$ nonfat milk diluted in Tris-buffered saline containing $0.05 \%$ Tween- 20 was used to block the membrane. Then, the membranes were incubated with primary antibodies to E-cadherin, N-cadherin, Vimentin, Bax, Bcl-2, MMP-9 or GAPDH (Abcam, Cambridge, MA, USA) overnight, followed by probing with an HRP-conjugated secondary antibody (Cell Signalling Technologies, Danvers, MA, USA) for $2 \mathrm{~h}$. The bound antibodies were detected using an enhanced chemiluminescence kit (Beyotime).

\section{Statistical analysis}

All statistical tests were conducted by using GraphPad Prism 5.01 software (GraphPad Software, Inc., La Jolla, CA, USA) and SPSS 19.0 statistical software package (IBM, Armonk, NY, USA). All experimental results from three independent experiments were expressed as mean \pm standard deviation (SD) and analyzed using Student's $t$-test. Survival analysis was calculated by Kaplan-Meier method with the log-rank test. Fisher's exact test was applied to evaluate the links between PANDAR and clinicopathological parameters. Survival data were analyzed using univariate and multivariate Cox regression methods. A $P$-value $<0.05$ was considered statistically significant.

\section{Results \\ PANDAR is upregulated in CCA clinical samples and cell lines}

First, we explored the relative expression of PANDAR in tumor and adjacent noncancerous tissues of patients with CCA. As shown in Figure 1A, the relative expression of PANDAR in tumor tissues from patients with CCA was 

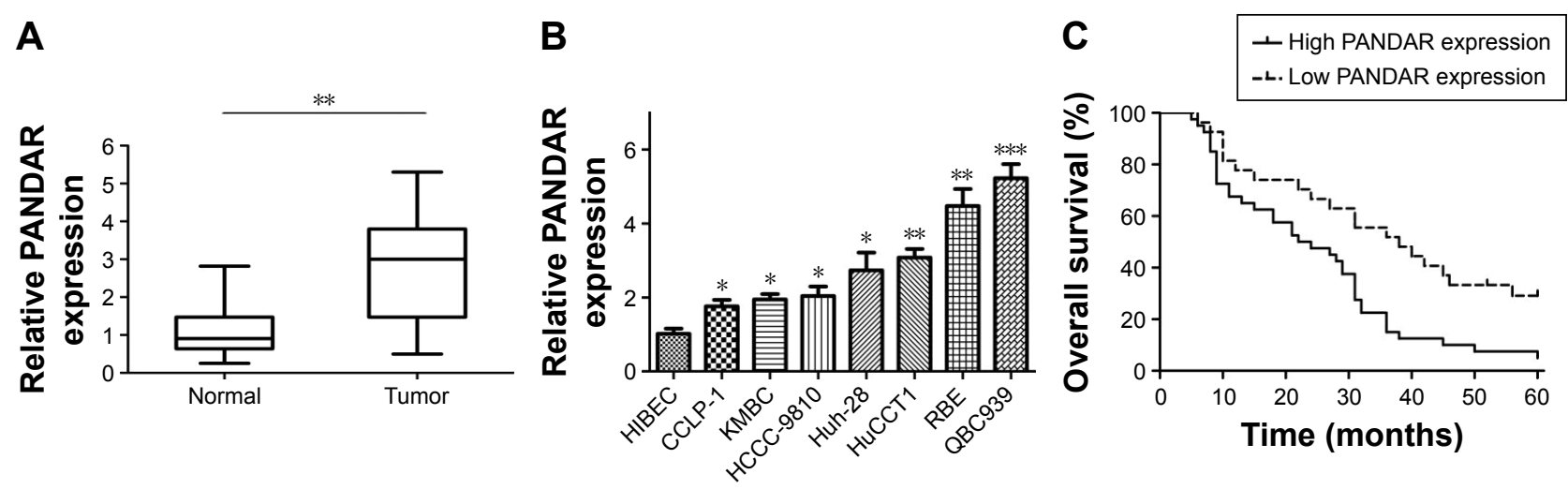

Figure I Relative expression of PANDAR in CCA and its clinical significance.

Notes: (A) Relative expression of PANDAR in CCA tissues and adjacent nontumor tissues were determined by qRT-PCR; (B) Relative expression of PANDAR in HIBEC and CCA cell lines were determined by qRT-PCR; (C) Kaplan-Meier survival curves showed that overexpression of PANDAR decreased overall survival in patients with CCA. $* p<0.05, * * p<0.01$, $* * * p<0.001$.

Abbreviations: CCA, cholangiocarcinoma; $\mathrm{QRT}-\mathrm{PCR}$, quantitative real-time PCR.

remarkably increased compared with the corresponding nontumor tissue by qRT-PCR. The expression of PANDAR in CCA cell lines including KMBC, HCCC-9810, HuCCT1, CCLP-1, Huh-28, QBC939 and RBE was also higher than that of HIBEC (Figure 1B).

\section{PANDAR overexpression is correlated with poor prognosis of patients with CCA} To verify the clinical significance of PANDAR in CCA, we evaluated the correlation of PANDAR expression with the clinicopathological characteristics of patients with CCA. As shown in Table 1, the high PANDAR group patients showed a more frequent lymph node invasion $(P=0.004)$, advanced TNM stage $(P=0.034)$ and more postoperative recurrence $(P=0.006)$. However, the other parameters, such as patients' gender, age and tumor site, were not associated with the expression of PANDAR. Moreover, Kaplan-Meier overall survival analysis and the log-rank test showed that patients with CCA with higher PANDAR expression had the worse overall survival after surgical procedure (Figure 1C). The univariate Cox regression analyses of overall survival showed that the lymph node invasion $(P=0.023)$, TNM stage $(P=0.020)$, postoperative recurrence $(P=0.004)$ and PANDAR expression $(P=0.005)$ were good prognostic indicators. Furthermore, multivariate Cox regression analyses confirmed the expression of PANDAR as an independent predictor for overall survival in patients with CCA $(P=0.041$, Table 2).

\section{PANDAR depletion inhibits cell proliferation}

To shed light on the roles of PANDAR in CCA, first, three siRNA targeting PANDAR and a scrambled nucleotide were transfected into QBC939 and RBE cells separately because the two cell lines expressed highest levels of PANDAR. Then, transfection analysis by flow cytometry demonstrated that the transfection efficiency of the fluorescence-labeled siRNA and Lipofectamine 3000 transfection reagent mixture reached $70 \%-90 \%$ in the two selected cell lines (Figure 2A). At $48 \mathrm{~h}$ after incubation, the relative expression of PANDAR was detected by qRT-PCR, and the results revealed a dramatic decline of PANDAR in all the three siRNAs targeting PANDAR compared to si-NC groups (Figure 2B). Among these, si-PANDAR-2 got the most powerful knockdown efficiency, and thus was chosen for subsequent studies. Compared to the negative control, transfection with PANDAR siRNA resulted in a remarkably restrained QBC939 and RBE cell viability as monitored by CCK-8 proliferation assay (Figure 2C). Moreover, clonogenic assay was conducted to probe the effect of PANDAR on anchorindependent growth of QBC939 and RBE cells. As shown in Figure 2D, CCA cells transfected with si-PANDAR-2 significantly abrogated the formative colony numbers.

\section{Knockdown of PANDAR increases apoptosis of CCA cells}

Flow cytometric analysis clarified that silencing of PANDAR induced apoptosis of CCA cells (Figure 3A). AO/EB double fluorescence staining assay showed similar results subsequently (Figure 3B). Next, we examined the activity of caspase-3 and caspase-9 in response to PANDAR. According to the results, PANDAR knockdown increased the expression of caspase-3 and caspase-9 in CCA cells (Figure 3C). Western blot analysis showed that silencing of PANDAR restrained the expression of Bcl-2 and increased the expression of Bax (Figure 3D). 
Table I Association between PANDAR expression and clinicopathological characteristics of CCA

\begin{tabular}{|c|c|c|c|c|}
\hline \multirow[t]{2}{*}{$\begin{array}{l}\text { Clinicopathological } \\
\text { features }\end{array}$} & \multirow[t]{2}{*}{$\begin{array}{l}\text { No of } \\
\text { patients (n) }\end{array}$} & \multicolumn{2}{|c|}{$\begin{array}{l}\text { PANDAR } \\
\text { expression }\end{array}$} & \multirow[t]{2}{*}{$P$-value } \\
\hline & & High (n) & Low $(n)$ & \\
\hline Gender & & & & 0.457 \\
\hline Male & 29 & 19 & 10 & \\
\hline Female & 38 & 21 & 17 & \\
\hline Age in years & & & & 0.307 \\
\hline$<60$ & 41 & 22 & 19 & \\
\hline$\geq 60$ & 26 & 18 & 8 & \\
\hline Tumor site & & & & 0.382 \\
\hline Intrahepatic & 13 & 7 & 6 & \\
\hline Perihilar & 30 & 16 & 14 & \\
\hline Distal & 24 & 17 & 7 & \\
\hline Tumor stage & & & & 0.799 \\
\hline $\mathrm{TI}-2$ & 24 & 15 & 9 & \\
\hline T3-4 & 43 & 25 & 18 & \\
\hline Lymph node invasion & & & & 0.004 \\
\hline Present & 44 & 32 & 12 & \\
\hline Absent & 23 & 8 & 15 & \\
\hline Vascular invasion & & & & 0.794 \\
\hline Positive & 21 & 12 & 9 & \\
\hline Negative & 46 & 28 & 18 & \\
\hline TNM stage & & & & 0.034 \\
\hline I-II & 15 & 5 & 10 & \\
\hline III-IV & 52 & 35 & 17 & \\
\hline Histology type & & & & 0.142 \\
\hline Papillary & 9 & 3 & 6 & \\
\hline Nonpapillary & 58 & 37 & 21 & \\
\hline Differentiation grade & & & & 0.602 \\
\hline Well/moderately & 22 & 12 & 10 & \\
\hline Poorly/undifferentiated & 45 & 28 & 17 & \\
\hline Postoperative recurrence & & & & 0.006 \\
\hline Present & 52 & 36 & 16 & \\
\hline Absent & 15 & 4 & II & \\
\hline Serum CEA level & & & & 0.193 \\
\hline$>5 \mathrm{ng} / \mathrm{mL}$ & 44 & 29 & 15 & \\
\hline$\leq 5 \mathrm{ng} / \mathrm{mL}$ & 23 & 11 & 12 & \\
\hline Serum CAI9-9 level & & & & 0.221 \\
\hline$>37 \mathrm{U} / \mathrm{mL}$ & 53 & 34 & 19 & \\
\hline$\leq 37 \mathrm{U} / \mathrm{mL}$ & 14 & 6 & 8 & \\
\hline HBV infection & & & & 0.788 \\
\hline Positive & 19 & 12 & 7 & \\
\hline Negative & 48 & 28 & 20 & \\
\hline
\end{tabular}

Abbreviations: CEA, carcinoembryonic antigen; CA19-9, carbohydrate antigen 19-9; HBV, hepatitis B virus.

\section{Silenced PANDAR inhibits metastasis in vitro and affects EMT in CCA cells}

To estimate the extent of PANDAR function in migration and invasion of CCA, we introduced wound healing assay and Transwell assay for detection. Interestingly, cells transfected with selected siRNA specifically targeting PANDAR led to less wound closure area than si-NC group (Figure 4A). Moreover, Transwell assay demonstrated that the migration capacity as well as invasive potential of the two selected cells was strikingly impaired due to PANDAR depletion (Figure 4B and C). Furthermore, MMP-9 was downregulated after silencing of PANDAR as seen by western blot analysis (Figure 4D). EMT is a vital mechanism associated with cell migration and invasion. Hence, we detected the expression of EMT-related proteins in CCA cells after transfection with corresponding siRNA. According to our results of western blot assay, the expression levels of $\mathrm{N}$-cadherin and Vimentin examined were strikingly decreased, whereas the expression of E-cadherin was boosted followed by siPANDAR (Figure 4E).

\section{Discussion}

During the past decades, the worldwide incidence of CCA has increased, especially in Asian countries., ${ }^{2,21,22}$ In addition, CCA is widely known to be an aggressive malignancy of the biliary duct that shows high mortality rate typically due to its late presentation and limited treatment measures. ${ }^{23,24}$ Therefore, the detection of specific therapeutic targets as well as identification of new diagnostic and prognostic biomarkers is the need of the hour for patients with CCA. lncRNAs were mistaken for nonfunctional secondary product of transcription for ages. During the past few years, lncRNAs were shown to be vital elements in cell biology and human diseases, especially in various cancers. Numerous evidences indicate that lncRNAs have imperative roles in various cancer-related processes including tumorigenesis, metastasis and angiogenesis. ${ }^{25-27}$ However, studies regarding the association between lncRNAs and CCA are scarce. Therefore, in this study, we focused on the novel cancer-related lncRNA PANDAR, which has got much attention in recent years and may provide new insights into CCA.

In this study, we found that PANDAR expression was upregulated not only in CCA tissue samples but also in CCA cell lines, which is in line with all of the digestive system neoplasms studied to date. ${ }^{17,18,20} \mathrm{We}$ further explored the correlation of expression of PANDAR with clinicopathological features of patients with CCA and the data showed that higher PANDAR expression was closely associated with lymph node invasion, advanced TNM stage and postoperative recurrence. The patients with higher PANDAR expression had statistically shorter survival time than those with lower PANDAR expression (by Kaplan-Meier analysis). Further multivariate analysis confirmed that PANDAR serves as an independent indicator for patients' overall survival. Taken together, the results of this study suggests that PANDAR is an oncogenic player or a predictor of overall survival for CCA.

Recent studies indicated that PANDAR was upregulated and led to cell growth in multiple cancers, such as gastric 
Table 2 Univariate and multivariate analysis of prognostic factors for overall survival in patients with CCA

\begin{tabular}{|c|c|c|c|c|c|c|}
\hline \multirow[t]{2}{*}{ Variables } & \multicolumn{3}{|c|}{ Univariate analysis } & \multicolumn{3}{|c|}{ Multivariate analysis } \\
\hline & $\mathbf{H R}$ & $95 \% \mathrm{Cl}$ & $P$-value & HR & $95 \% \mathrm{Cl}$ & $P$-value \\
\hline \multicolumn{7}{|l|}{ Overall survival } \\
\hline Gender (male vs female) & 1.085 & $0.643-1.832$ & 0.759 & & & \\
\hline Age $(\geq 60$ vs $<60$ years $)$ & 1.063 & $0.626-1.805$ & 0.822 & & & \\
\hline Tumor site (extrahepatic vs intrahepatic) & 1.034 & $0.535-2.000$ & 0.920 & & & \\
\hline Tumor stage (T3-4 vs TI-2) & 1.433 & $0.824-2.490$ & 0.202 & & & \\
\hline Lymph node invasion (positive vs negative) & 1.953 & $1.096-3.480$ & 0.023 & & & 0.189 \\
\hline Vascular invasion (positive vs negative) & 1.341 & $0.773-2.328$ & 0.296 & & & \\
\hline TNM stage (III-IV vs I-II) & 2.36 & I.147-4.859 & 0.020 & & & 0.307 \\
\hline Histology type (papillary vs nonpapillary) & 0.616 & $0.264-1.440$ & 0.264 & & & \\
\hline Differentiation grade (poorly/undifferentiated vs well/moderately) & 1.748 & $0.987-3.098$ & 0.056 & & & 0.145 \\
\hline Postoperative recurrence (present vs absent) & 2.903 & I.409-5.982 & 0.004 & 2.433 & I.157-5.118 & 0.019 \\
\hline Serum CEA level $(>5 \mathrm{ng} / \mathrm{mL}$ vs $\leq 5 \mathrm{ng} / \mathrm{mL})$ & 1.368 & $0.781-2.396$ & 0.273 & & & \\
\hline Serum CA19-9 level ( $>37 \mathrm{U} / \mathrm{mL}$ vs $\leq 37 \mathrm{U} / \mathrm{mL})$ & 1.475 & $0.761-2.859$ & 0.250 & & & \\
\hline HBV infection (positive vs negative) & 1.426 & $0.806-2.521$ & 0.223 & & & \\
\hline PANDAR expression (high vs low) & 2.228 & $1.269-3.913$ & 0.005 & 1.830 & $1.026-3.264$ & $0.04 I$ \\
\hline
\end{tabular}

Note: Data in bold indicates statistical significance at $P<0.05$.

Abbreviations: CA19-9, carbohydrate antigen 19-9; CEA, carcinoembryonic antigen; HBV, hepatitis B virus.

cancer, hepatocellular carcinoma, bladder carcinoma, colon cancer and breast cancer. ${ }^{17-20,28}$ A recent study indicated that PANDAR acts as a tumor-promoter in breast cancer by regulating G1/S transition. PANDAR-mediated promotion of cell proliferation occurs via regulation of $\mathrm{p} 16^{\mathrm{INK} 4 \mathrm{~A}} \cdot{ }^{28}$ In order

A
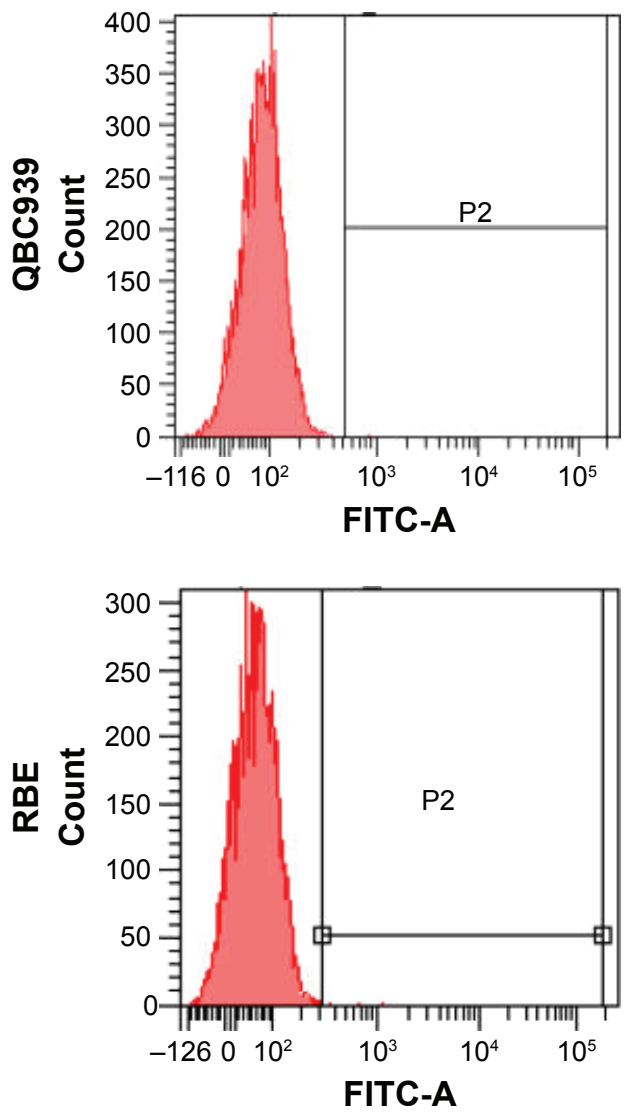

to understand the biological functions of PANDAR in CCA, we examined the differences in cell proliferation after suppressing PANDAR expression by using CCK-8 and colony formation assays. The results showed that cell viability and colony formation capacity were both abrogated in the

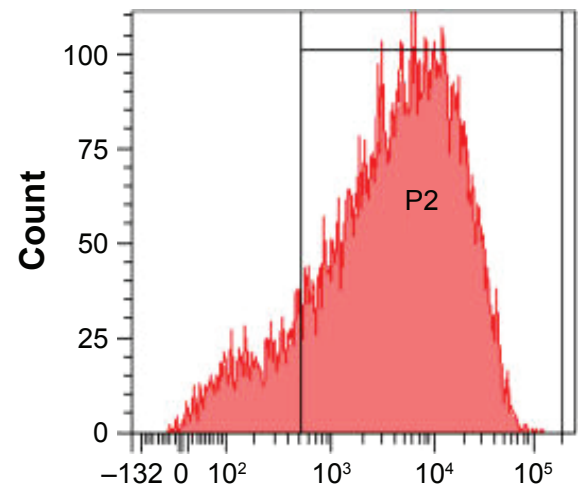

$86.9 \%$

FITC-A

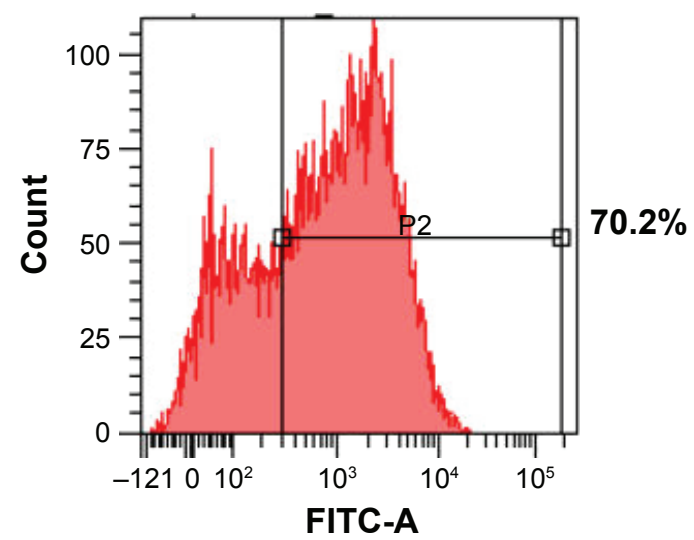

Figure 2 (Continued) 
B

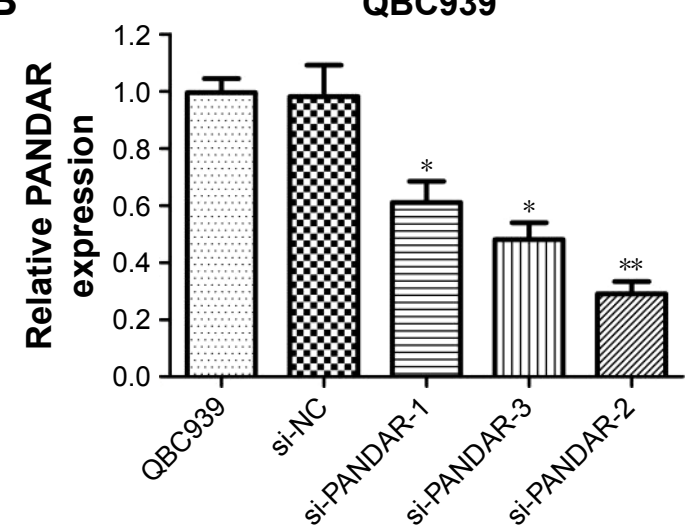

C

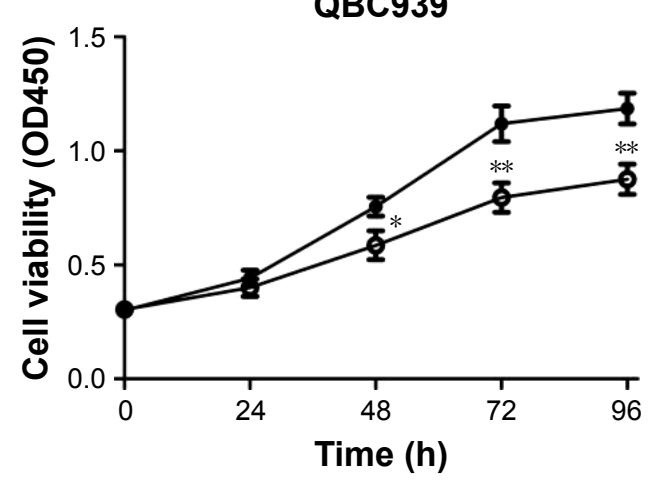

RBE

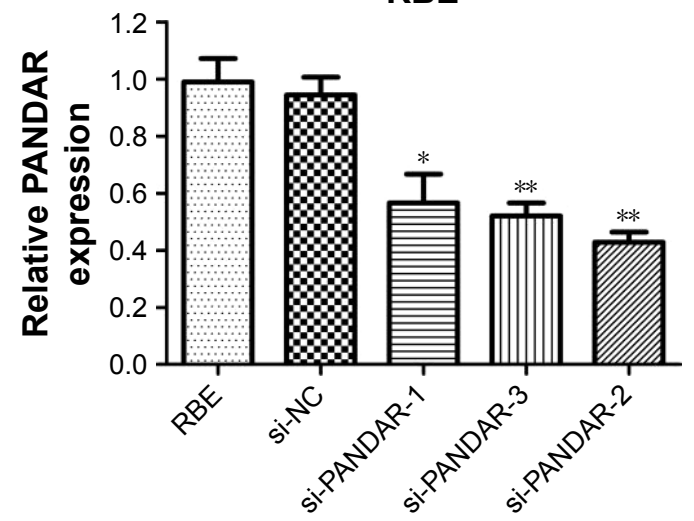

RBE

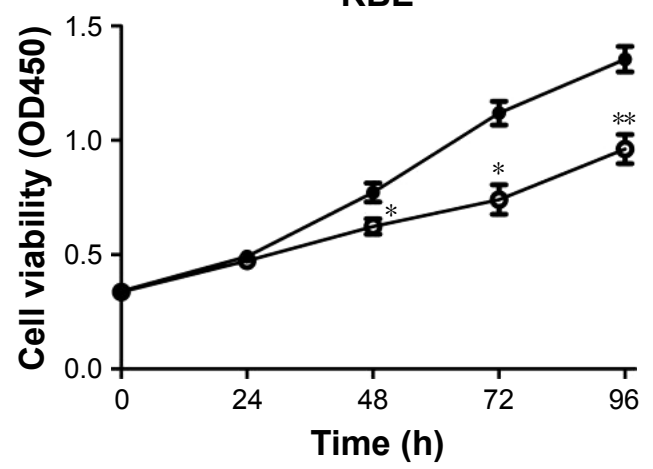

si-NC $\odot$ si-PANDAR-2

D

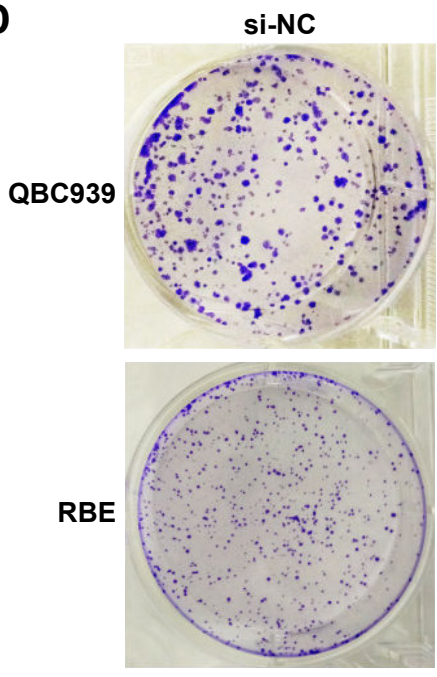

si-PANDAR-2

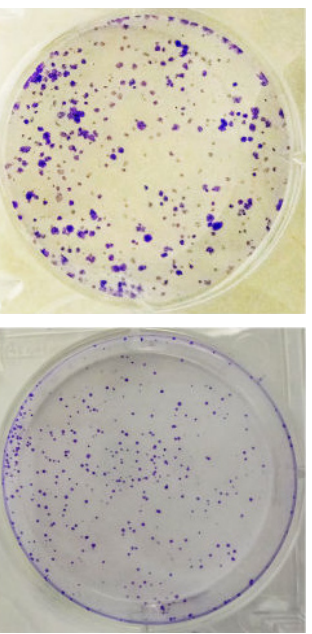

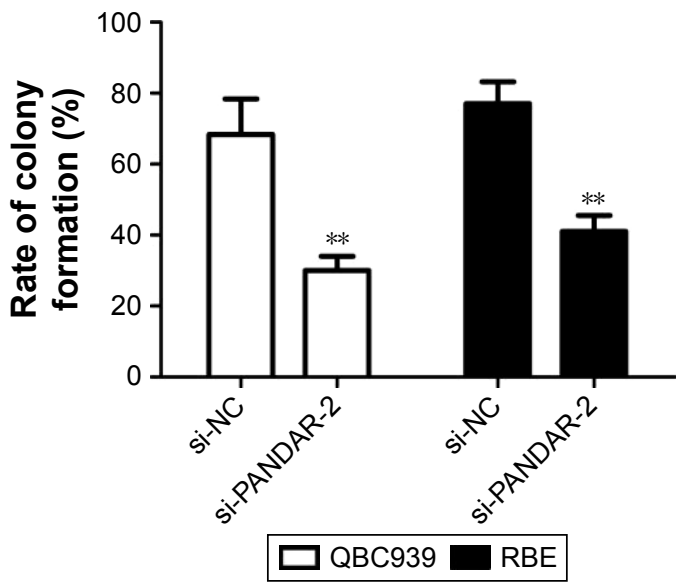

Figure 2 Transfection and knockdown efficiency of PANDAR-specific siRNAs and PANDAR depletion retarded cell proliferation and colony formation in CCA cells. Notes: (A) Transfection efficiency detected by flow cytometry in QBC939 and RBE cells; (B) Relative expression of PANDAR in QBC939 and RBE cells was significantly decreased by three siRNAs specifically targeting PANDAR compared with si-NC; (C) CCK-8 assay showed that silenced PANDAR inhibited cell proliferation of QBC939 and RBE cells; (D) Clonogenic assay showed that silenced PANDAR dramatically impaired the colony formation ability of $Q B C 939$ and $R B E$ cells. $* P<0.05$, $* * P<0.01$. Abbreviation: CCA, cholangiocarcinoma.

PANDAR knockdown groups. Apoptosis signaling can be activated by receptor-mediated pathway and mitochondrial pathway and both of the pathways are correlated with the regulation of caspase-3, caspase-9, Bcl-2 and Bax. In our study, flow cytometric analysis as well as AO/EB staining assay showed that PANDAR acts as a defender against cancer apoptosis, and apoptosis-related factors (caspase-3 and caspase-9) were both activated analyzed by caspases detection. Furthermore, Bcl-2 was downregulated after PANDAR knockdown, whereas Bax was activated followed 
A
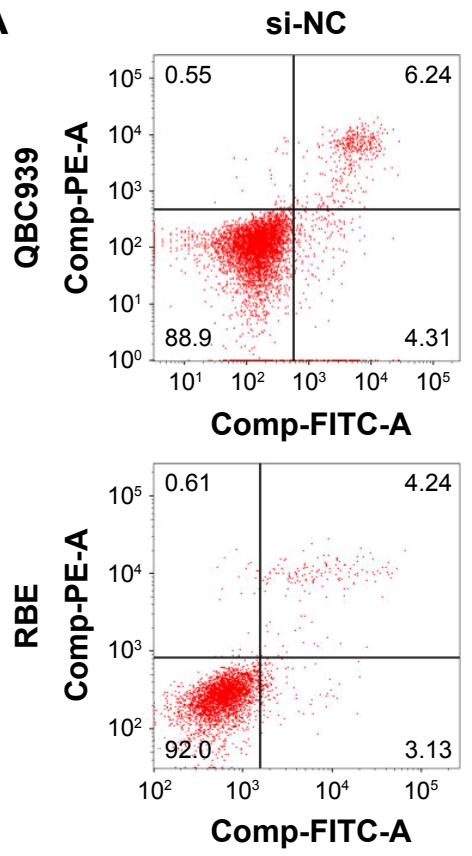

B
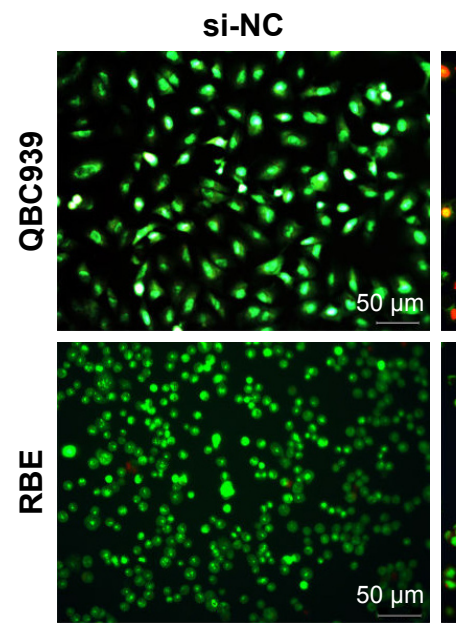

C

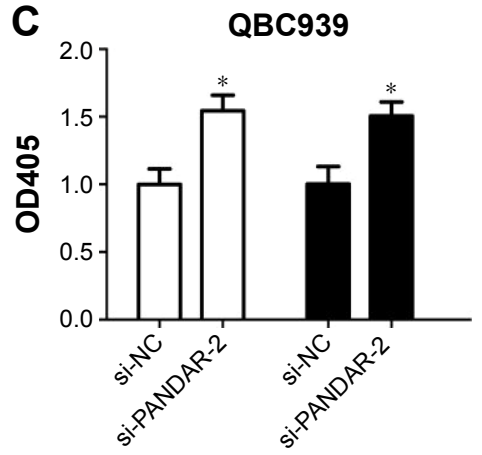

Si-PANDAR-2
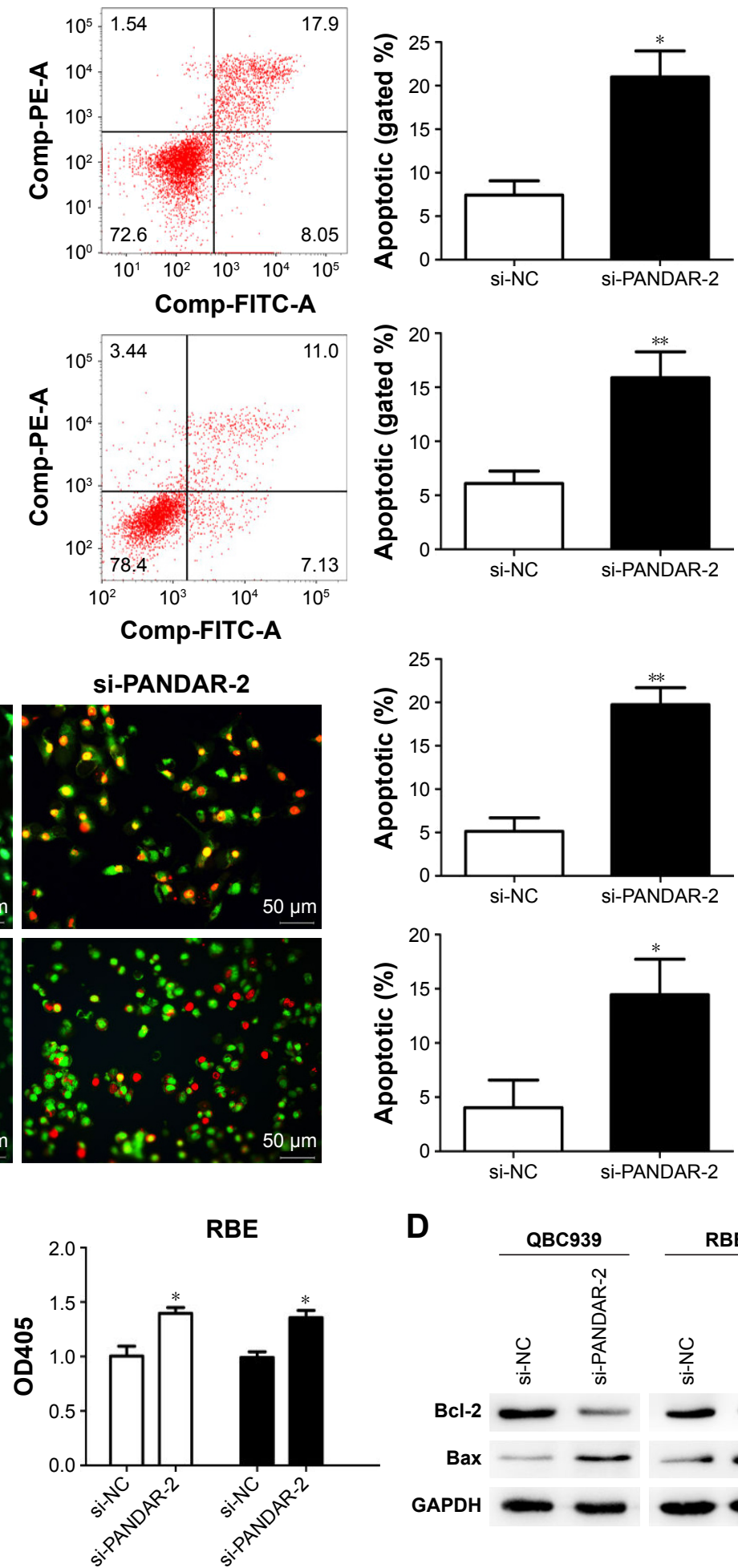

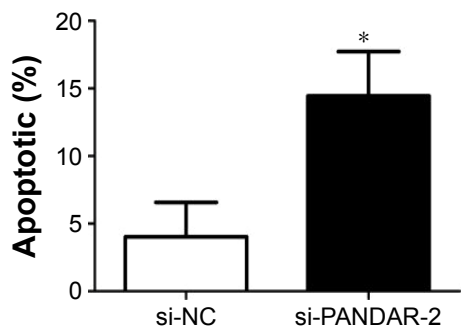

D

Si-PANDAR-2
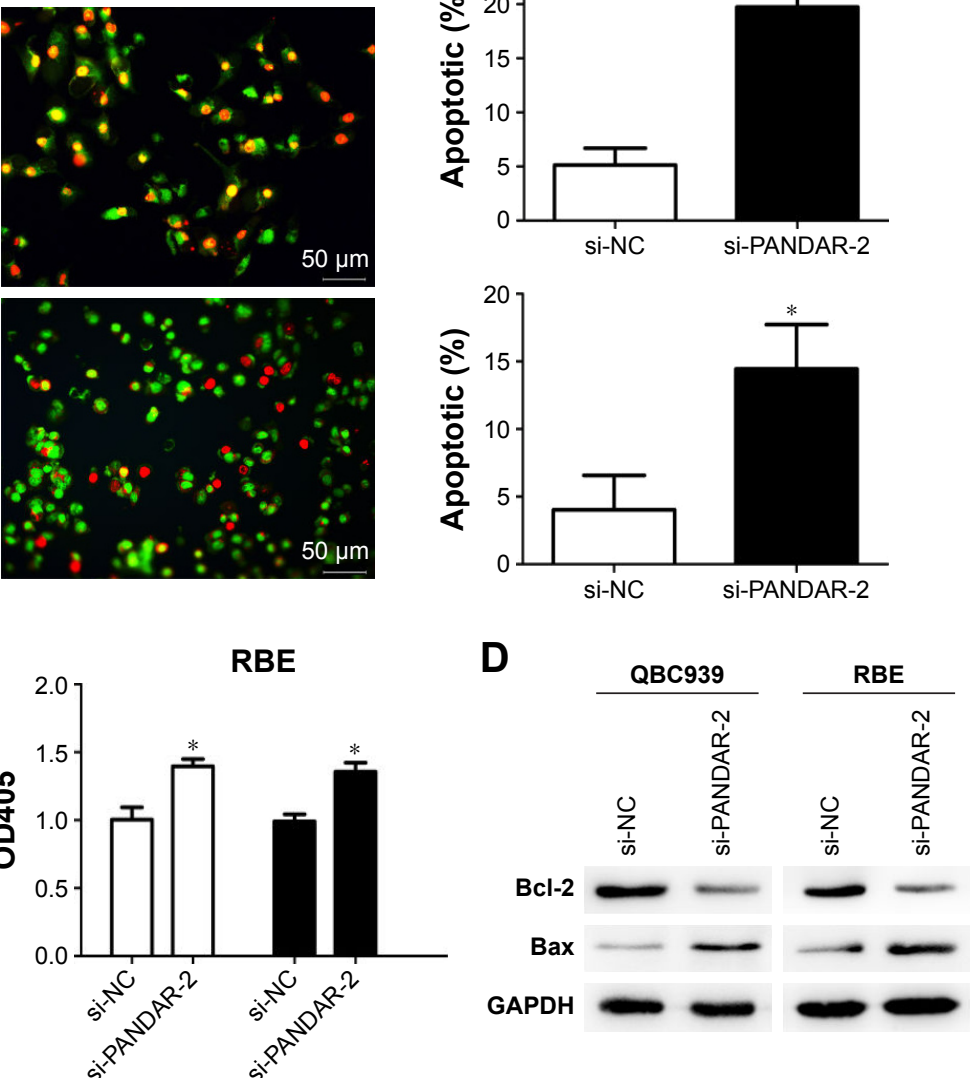

Caspase-3

Caspase-9

Figure 3 PANDAR depletion increased apoptosis in CCA cells.

Notes: (A) Flow cytometry for apoptosis analysis showed that silenced PANDAR significantly promoted apoptosis of QBC939 and RBE cells compared to negative control siRNA; (B) AO/EB double fluorescence staining assay showed that silenced PANDAR significantly promoted apoptosis of QBC939 and RBE cells compared to negative control siRNA ( $\times 200)$; (C) Relative expression of caspase-3 and caspase-9 in QBC939 and RBE cells were activated by si-PANDAR-2; (D) Western blot analysis showed that knockdown of PANDAR restrained the expression of $\mathrm{Bcl}-2$ and increased Bax expression in QBC939 and RBE cells. $* P<0.05$, $* * P<0.01$.

Abbreviations: CCA, cholangiocarcinoma; $A O / E B$, acridine orange/ethidium bromide. 


\section{A $\quad$ QBC939}

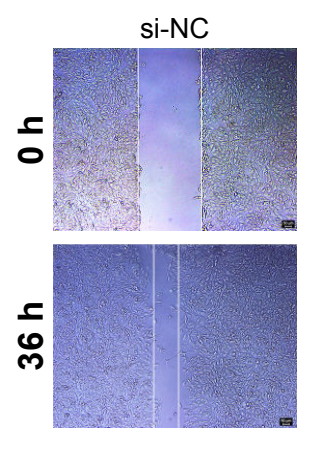

\section{RBE}

si-PANDAR-2

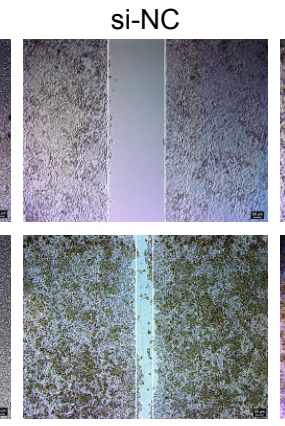

si-PANDAR-2

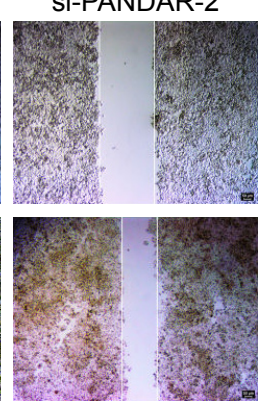

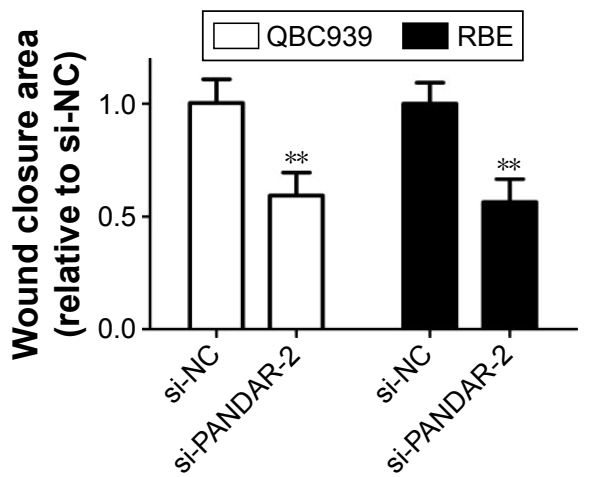

B
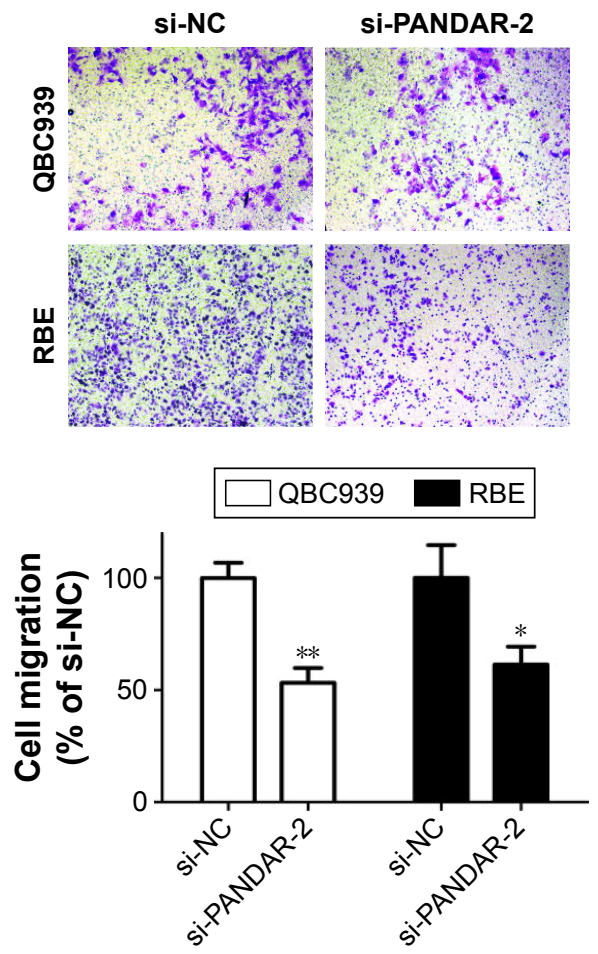

D

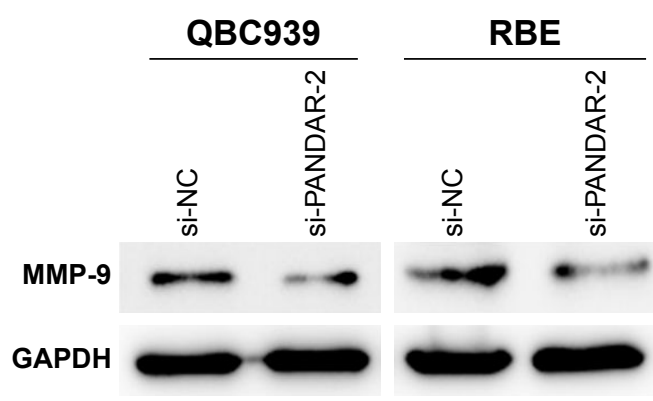

C
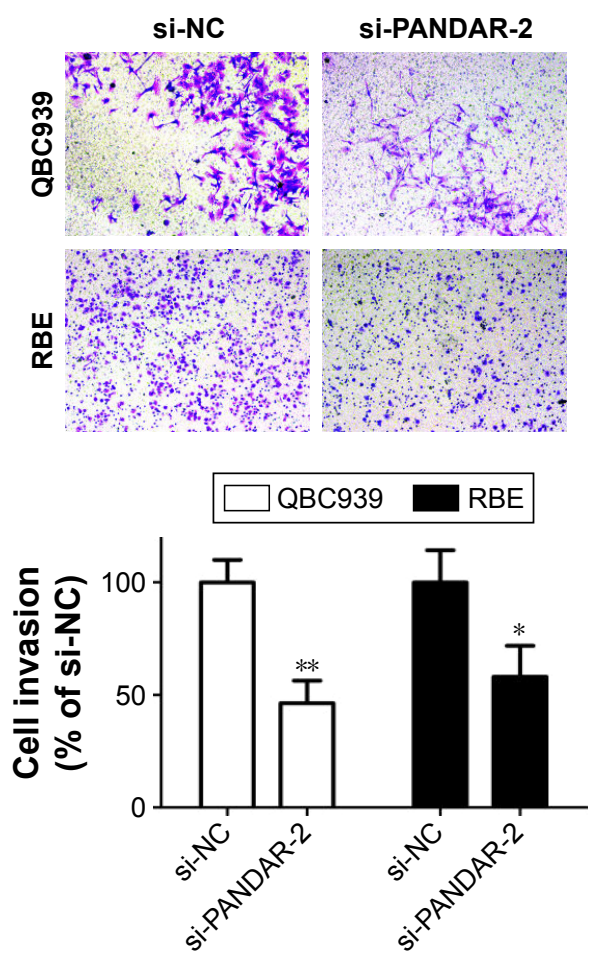

E

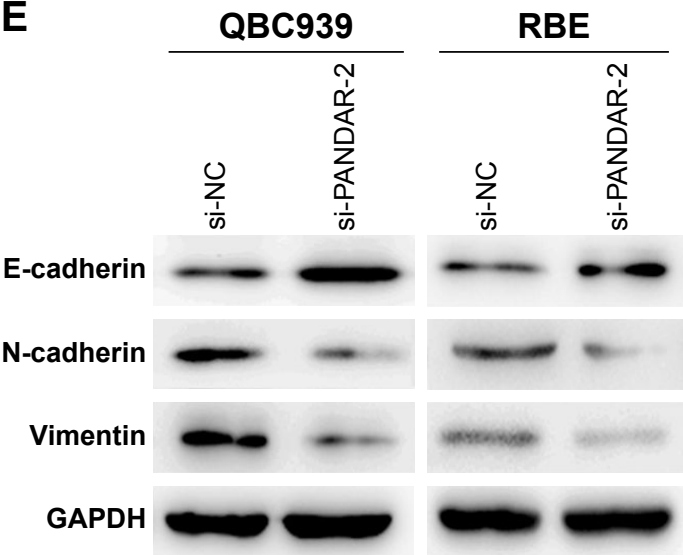

Figure 4 Knockdown of PANDAR impaired migration and invasion capacity in vitro and reversed EMT of CCA cells.

Notes: (A) Wound healing assay showed that silenced PANDAR inhibited migration ability of QBC939 and RBE cells $(\times 50)$; (B) Transwell migration assay showed that silenced PANDAR inhibited migration capacity of QBC939 and RBE cells $(\times 100)$; (C) Transwell invasion assay showed that silenced PANDAR inhibited invasion potential of QBC939 and RBE cells (×100); (D) Western blot analysis showed that knockdown of PANDAR restrained the expression of MMP-9 in QBC939 and RBE cells; (E) Knockdown of PANDAR reversed EMT in QBC939 and RBE cells. $* P<0.05, * * P<0.01$.

Abbreviations: CCA, cholangiocarcinoma; EMT, epithelial-to-mesenchymal transition. 
by silencing of PANDAR. These results suggest that Bcl-2/ caspase-3 pathway act by mediating apoptosis induced by the downregulation of PANDAR.

Next, we performed Transwell and western blot assays to explore whether expression of PANDAR affects migration capacity and invasive potential of CCA cells in vitro and investigate its associations with MMP-9. Transwell assay showed that siRNA targeting PANDAR significantly reduced the migration and invasion of CCA cells. MMP-9 has been proved to facilitate the invasion capacity of cancer cells by damaging the histological barrier around the extracellular matrix. ${ }^{29}$ In this study, we found that MMP-9 was decreased followed by the knockdown of PANDAR. These results demonstrate that PANDAR facilitates invasion potential partly by upregulating MMP-9 expression. EMT, a major mechanism involved in cell invasiveness, was proven to be involved in tumor metastasis, and the characteristics of EMT included the lost E-cadherin and enhanced N-cadherin and Vimentin expression. ${ }^{30-32}$ It has been reported that LINC01133 inhibits EMT and metastasis in colorectal carcinoma via binding to SRSF6 as a target mimic. ${ }^{33}$ In addition, overexpressed MALAT1 in tongue cancer activated EMT and restrained cell apoptosis by regulating Wnt/beta-catenin signaling pathway. ${ }^{34}$ For CCA, E-cadherin and N-cadherin switch promotes cell progression through TGF-beta-induced EMT, which suggested a target for illuminating the mechanisms of invasion and metastasis in CCA. ${ }^{35}$ In this study, western blot results showed reversed EMT markers after knockdown of PANDAR, which indicates that PANDAR is involved in mechanisms related to the facilitation of CCA cell migration and invasion, and impaired the potential of metastasis in vitro partly by regulating the EMT pathway.

In our study, there are still some limitations to come to an incontrovertible conclusion. CCA is a multifactorial, multistep, aggressive disease with various genes activated or silenced. Moreover, the molecular mechanisms of lncRNAs seem to be highly diverse and act at miscellaneous levels to manipulate cancer development and progression. Hence, further studies are needed to unravel the precise mechanism behind the altered expression of PANDAR in CCA.

\section{Conclusion}

We first confirmed that PANDAR was statistically overexpressed in CCA and therefore might be a potential indicator of a negative prognosis for patients with CCA. Moreover, downregulation of PANDAR inhibited the proliferation, migration, invasion and EMT of CCA cells and facilitate its apoptosis. In summary, these results indicate that PANDAR plays oncogenic roles in CCA and serves as a potential prognostic biomarker and therapeutic target for treatment of CCA.

\section{Acknowledgments}

This study was funded by the National Natural Science Foundation of China (Grant Nos 81602088 and 81170426), Health and Family Planning Commission Research Project of Heilongjiang Province (Grant No 2016-049), Heilongjiang Postdoctoral Science Foundation (Grant No LBH-Z16096), Postgraduate innovative research project of Harbin Medical University (Grant Nos YJSCX2016-21HYD), Innovative Science Foundation of Harbin Medical University (Grant No 2016LCZX09) and Natural Science Foundation of Heilongjiang Province (Grant Nos H201396). The funders had no role in study design, data collection and analysis, decision to publish or preparation of the manuscript.

\section{Disclosure}

The authors report no conflicts of interest in this work.

\section{References}

1. DeOliveira ML, Cunningham SC, Cameron JL, et al. Cholangiocarcinoma: thirty-one-year experience with 564 patients at a single institution. Ann Surg. 2007;245(5):755-762.

2. Khan SA, Davidson BR, Goldin RD, et al; British Society of Gastroenterology. Guidelines for the diagnosis and treatment of cholangiocarcinoma: an update. Gut. 2012;61(12):1657-1669.

3. Aljiffry M, Walsh MJ, Molinari M. Advances in diagnosis, treatment and palliation of cholangiocarcinoma: 1990-2009. World $J$ Gastroenterol. 2009;15(34):4240-4262.

4. Skipworth JR, Olde Damink SW, Imber C, Bridgewater J, Pereira SP, Malagó M. Review article: surgical, neo-adjuvant and adjuvant management strategies in biliary tract cancer. Aliment Pharmacol Ther. 2011; 34(9): 1063-1078.

5. Alberts SR, Gores GJ, Kim GP, et al. Treatment options for hepatobiliary and pancreatic cancer. Mayo Clin Proc. 2007;82(5):628-637.

6. Carninci P, Kasukawa T, Katayama S, et al; FANTOM Consortium; RIKEN Genome Exploration Research Group and Genome Science Group (Genome Network Project Core Group). The transcriptional landscape of the mammalian genome. Science. 2005;309(5740): $1559-1563$.

7. Zhang A, Zhang J, Kaipainen A, Lucas JM, Yang H. Long non-coding RNA: a newly deciphered "code" in prostate cancer. Cancer Lett. 2016; 375(2):323-330.

8. Raveh E, Matouk IJ, Gilon M, Hochberg A. The H19 long non-coding RNA in cancer initiation, progression and metastasis - a proposed unifying theory. Mol Cancer. 2015;14:184.

9. Schmitt AM, Chang HY. Long noncoding RNAs in cancer pathways. Cancer Cell. 2016;29(4):452-463.

10. Walsh AL, Tuzova AV, Bolton EM, Lynch TH, Perry AS. Long noncoding RNAs and prostate carcinogenesis: the missing 'linc'? Trends Mol Med. 2014;20(8):428-436.

11. Malek E, Jagannathan S, Driscoll JJ. Correlation of long non-coding RNA expression with metastasis, drug resistance and clinical outcome in cancer. Oncotarget. 2014;5(18):8027-8038. 
12. Gutschner T, Diederichs $S$. The hallmarks of cancer: a long non-coding RNA point of view. RNA Biol. 2012;9(6):703-719.

13. Gupta RA, Shah N, Wang KC, et al. Long non-coding RNA HOTAIR reprograms chromatin state to promote cancer metastasis. Nature. 2010;464(7291):1071-1076.

14. Kotake Y, Nakagawa T, Kitagawa K, et al. Long non-coding RNA ANRIL is required for the PRC2 recruitment to and silencing of $\mathrm{p} 15$ (INK4B) tumor suppressor gene. Oncogene. 2011;30(16):1956-1962.

15. Wang WT, Ye H, Wei PP, et al. LncRNAs H19 and HULC, activated by oxidative stress, promote cell migration and invasion in cholangiocarcinoma through a ceRNA manner. J Hematol Oncol. 2016;9(1):117.

16. Hung T, Wang Y, Lin MF, et al. Extensive and coordinated transcription of noncoding RNAs within cell-cycle promoters. Nat Genet. 2011; 43(7):621-629.

17. Ma P, Xu T, Huang M, Shu Y. Increased expression of LncRNA PANDAR predicts a poor prognosis in gastric cancer. Biomed Pharmacother. 2016;78:172-176.

18. Peng $\mathrm{W}$, Fan $\mathrm{H}$. Long non-coding RNA PANDAR correlates with poor prognosis and promotes tumorigenesis in hepatocellular carcinoma Biomed Pharmacother. 2015;72:113-118.

19. Zhan Y, Lin J, Liu Y, et al. Up-regulation of long non-coding RNA PANDAR is associated with poor prognosis and promotes tumorigenesis in bladder cancer. $J$ Exp Clin Cancer Res. 2016;35(1):83.

20. Lu M, Liu Z, Li B, Wang G, Li D, Zhu Y. The high expression of long non-coding RNA PANDAR indicates a poor prognosis for colorectal cancer and promotes metastasis by EMT pathway. J Cancer Res Clin Oncol. 2017;143(1):71-81.

21. Bergquist A, von Seth E. Epidemiology of cholangiocarcinoma. Best Pract Res Clin Gastroenterol. 2015;29(2):221-232.

22. Rizvi S, Gores GJ. Pathogenesis, diagnosis, and management of cholangiocarcinoma. Gastroenterology. 2013;145(6):1215-1229.

23. Zhu AX. Future directions in the treatment of cholangiocarcinoma Best Pract Res Clin Gastroenterol. 2015;29(2):355-361.

24. Ciombor KK, Goff LW. Current therapy and future directions in biliary tract malignancies. Curr Treat Options Oncol. 2013;14(3):337-349.
25. Wang F, Ni H, Sun F, Li M, Chen L. Overexpression of IncRNA AFAP1-AS1 correlates with poor prognosis and promotes tumorigenesis in colorectal cancer. Biomed Pharmacother. 2016;81:152-159.

26. Gao H, Song X, Kang T, et al. Long noncoding RNA CRNDE functions as a competing endogenous RNA to promote metastasis and oxaliplatin resistance by sponging miR-136 in colorectal cancer. Onco Targets Ther. 2017;10:205-216.

27. Ruan W, Wang P, Feng S, Xue Y, Li Y. Long non-coding RNA small nucleolar RNA host gene 12 (SNHG12) promotes cell proliferation and migration by upregulating angiomotin gene expression in human osteosarcoma cells. Tumour Biol. 2016;37(3):4065-4073.

28. Sang Y, Tang J, Li S, et al. LncRNA PANDAR regulates the G1/S transition of breast cancer cells by suppressing p16(INK4A) expression. Sci Rep. 2016;6:22366.

29. Folgueras AR, Pendás AM, Sánchez LM, López-Otín C. Matrix metalloproteinases in cancer: from new functions to improved inhibition strategies. Int J Dev Biol. 2004;48(5-6):411-424

30. Ye X, Weinberg RA. Epithelial-mesenchymal plasticity: a central regulator of cancer progression. Trends Cell Biol. 2015;25(11):675-686.

31. Wang LK, Pan SH, Chang YL, et al. MDA-9/Syntenin-Slug transcriptional complex promote epithelial-mesenchymal transition and invasion/ metastasis in lung adenocarcinoma. Oncotarget. 2016;7(1):386-401.

32. Yamada S, Fuchs BC, Fujii T, et al. Epithelial-to-mesenchymal transition predicts prognosis of pancreatic cancer. Surgery. 2013;154(5): 946-954.

33. Kong J, Sun W, Li C, et al. Long non-coding RNA LINC01133 inhibits epithelial-mesenchymal transition and metastasis in colorectal cancer by interacting with SRSF6. Cancer Lett. 2016;380(2):476-484.

34. Liang J, Liang L, Ouyang K, Li Z, Yi X. MALAT1 induces tongue cancer cells' EMT and inhibits apoptosis through Wnt/B-catenin signaling pathway. J Oral Pathol Med. 2017;46(2):98-105.

35. Araki K, Shimura T, Suzuki H, et al. E/N-cadherin switch mediates cancer progression via TGF- $\beta$-induced epithelial-to-mesenchymal transition in extrahepatic cholangiocarcinoma. Br J Cancer. 2011; 105(12):1885-1893.
OncoTargets and Therapy

\section{Publish your work in this journal}

OncoTargets and Therapy is an international, peer-reviewed, open access journal focusing on the pathological basis of all cancers, potential targets for therapy and treatment protocols employed to improve the management of cancer patients. The journal also focuses on the impact of management programs and new therapeutic agents and protocols on

\section{Dovepress}

patient perspectives such as quality of life, adherence and satisfaction. The manuscript management system is completely online and includes a very quick and fair peer-review system, which is all easy to use. Visit http://www.dovepress.com/testimonials.php to read real quotes from published authors. 